\title{
INTERAÇÃO GENÓTIPO X CICLOS DE COLHEITA DE CANA-DE-AÇÚCAR DA ZONA DA MATA NORTE DE PERNAMBUCO $\left({ }^{1}\right)$
}

\author{
LUIZ JOSÉ OLIVEIRA TAVARES DE MELO $\left({ }^{2}\right)$; FRANCISCO JOSÉ DE OLIVEIRA $\left({ }^{3}\right)$; \\ GERSON QUIRINO BASTOS $\left({ }^{3}\right)$; CLODOALDO JOSÉ DA ANUNCIAÇÃO FILHO $\left({ }^{3}\right)$; \\ ODEMAR VICENTE DOS REIS $\left({ }^{4}\right)$
}

\begin{abstract}
RESUMO
Este trabalho teve por objetivo estudar, na Região da Mata Norte de Pernambuco, o desempenho de clones RB da série 94 de cana-de-açúcar da Universidade Federal Rural de Pernambuco (UFRPE) quanto à produtividade agroindustrial, bem como avaliar as interações genótipo x ambiente e analisar a magnitude de alguns parâmetros genéticos. Os trabalhos experimentais foram desenvolvidos durante as safras agrícolas de 1999/2000, 2000/2001, 2001/2002 e 2002/2003, em área agrícola da Usina Olho D'Água, localizada na Zona Canavieira da Mata Norte de Pernambuco, no Município de Camutanga $\left(07^{\circ} 24^{\prime} \mathrm{S}\right.$ e $\left.35^{\circ} 16^{\prime} \mathrm{W}\right)$. O delineamento experimental foi o de blocos casualizados, com quatro blocos e dezesseis genótipos, sendo quatro deles variedades-padrão. Os resultados demonstraram que as variedades-padrão RB813804, RB72454, SP79-1011 e SP70-1143 confirmaram o potencial em produção de cana por hectare e de açúcar por hectare nas condições ambientais da Zona da Mata Norte de Pernambuco. O efeito de corte da cana foi altamente significativo, indicando o comportamento específico entre os genótipos nos diversos cortes da cana. A tonelada de cana por hectare foi o componente mais influenciado pelos ciclos de colheita da cana.
\end{abstract}

Palavras-chave: Saccharum spp., variedades, parâmetros genéticos, interação genótipo x ambiente.

\section{ABSTRACT \\ SUGARCANE GENOTYPE X HARVEST CYCLES INTERACTION IN ZONA DA MATA NORTE OF PERNAMBUCO}

This work aimed to study the development of sugarcane RB clones, series 94 from the Universidade Federal Rural de Pernambuco (UFRPE) in the North Forest Zone of Pernambuco, regarding to agroindustrial yield, evaluate genotype $x$ environment interactions and the magnitude of some genetic parameters. The experimental work was done during the agricultural years 1999/2000, 2000/2001, 2001/ 2002 and 2002/2003, in Olho d'Água sugar mill, located at the North Forest Sugar Cane Zone of Pernambuco, in Camutanga $\left(07^{\circ} 24^{\prime} \mathrm{S}\right.$ and $\left.35^{\circ} 16^{\prime} \mathrm{W}\right)$. The experimental design was randomized blocks with four replicates and sixteen genotypes, being four of them standard clones. As to cane and sugar yield per hectare, the standard clones RB 813804, RB 72454, SP 79-1011 and SP 70-1143 confirmed their potential. The effect of cane cut was highly significant, indicating specific behaviour among the genotypes. Yield per hectare was the component most influenced by cane cutting cycles.

Key words: Saccharum spp., varieties, genetic parameters, genotype x environment interaction.

$\left({ }^{1}\right)$ Extraído da dissertação de mestrado apresentada pelo primeiro autor à Universidade Federal Rural de Pernambuco (UFRPE). Recebido para publicação em 3 de dezembro de 2004 e aceito em 16 de novembro de 2005.

$\left({ }^{2}\right)$ Aluno do curso de Mestrado do Programa de Pós-Graduação em Agronomia "Melhoramento Genético em Plantas" da UFRPE. Autor correspondente. E-mail: luizjose@hotmail.com

$\left({ }^{3}\right)$ Departamento de Agronomia da UFRPE, Rua Dom Manoel de Medeiros s/n, Dois Irmãos, 52171-900, Recife (PE). Email: franseol@uol.com.br; bastosgq@hotmail.com; cjose@ufrpe.br.

$\left({ }^{4}\right)$ Empresa Pernambucana de Pesquisa Agropecuária. Av. San Martin 1371, Bongi, Caixa Postal 1022, 50761-000 Recife, PE. E-mail: ipa@ipa.br. 


\section{INTRODUÇÃO}

O Brasil é um dos mais tradicionais produtores de cana-de-açúcar cuja cultura é implantada em vários tipos de solos e sob influência de diferentes climas, o que resulta em diversos tipos de ambientes com as quais ela interage (DiAs, 1997).

No país, existe número relativamente grande de variedades obtidas em programas de melhoramento locais ou introduzidas de outras regiões, que são avaliadas continuamente na experimentação, visando determinar o comportamento e a viabilidade de seu aproveitamento em plantios comerciais. Usa-se, dessa maneira, indicar as mais adaptadas à cada microrregião produtora, possibilitando, assim a racionalização através da utilização de variedades superiores em produtividade, sem desconsiderar outras importantes características agroindustriais. Desse modo, tenta-se contornar o problema da queda de produção dada à degenerescência relacionada a aspectos fitossanitários BAssinelo et al. (1984). Para GHeller et al. (1996), a liberação contínua de novas variedades de cana-deaçúcar pelos programas de melhoramento dessa lavoura, garante ao plantador ganhos em produtividade agrícola e industrial, o que recomenda a substituição de variedade a cada reforma do canavial.

A seleção de clones com boas características agronômicas e tecnológicas tem sido perseguidas pelos programas de melhoramento de cana-de-açúcar no Brasil. Em tais programas os clones são avaliados em diferentes ambientes, antes da seleção final para recomendação e multiplicação na exploração comercial. Como os ambientes utilizados nos experimentos são bastante diversificados, espera-se que ocorra a interação entre clones e ambientes, com papel importante na manifestação fenotípica. Em razão da relevância desta interação, é necessário obter informações sobre sua magnitude e natureza, para permitir a escolha da melhor estratégia de seleção. Além disso, deve-se considerar que essa interação reduz a correlação genética e, por conseqüência, o ganho com a seleção (FALCONER e MACKAY 1996).

A avaliação da interação genótipos $x$ ambientes torna-se de grande importância no melhoramento, pois, no caso de sua existência, há possibilidade de o melhor genótipo em um determinado ambiente não o ser em outro (CRUZ e REGAZZI, 1997). Estudos a respeito da relação genótipo $x$ ambiente e os efeitos dessa interação têm sido objeto de diversas pesquisas em programas de melhoramento genético da cana-de-açúcar, a exemplo dos trabalhos realizados por FARIAS (1992), SOUZAVieira e Milligan (1999), Landell et al. (1999) e Silva et al. (2002b), entre outros.

Para RAmAlHo et al. (2001), o conhecimento da variabilidade decorrente das diferenças genéticas existentes, manifestadas pelos caracteres agronômicos nas populações e o quanto se deve a diferenças genéticas é de fundamental importância em qualquer programa de melhoramento, porque permite conhecer o controle genético do caráter e o potencial da população para seleção. Estudando as estimativas de alguns parâmetros genéticos em cana-de-açúcar, LANDELL et al. (1999), observaram que os caracteres tonelada de cana por hectare e tonelada de pol por hectare, a componente de variância clones $\mathrm{x}$ ambientes é elevada, confirmando a resposta específica de clones a ambientes diferenciados, com variações consideráveis de resposta à mudança de ambientes.

Este trabalho teve por objetivo estudar, na Região da Mata Norte de Pernambuco, o desempenho de clones RB da série 94 de cana-de-açúcar da Universidade Federal Rural de Pernambuco (UFRPE) quanto à produtividade agroindustrial, bem como avaliar as interações genótipo $\mathrm{x}$ ambiente e analisar a magnitude de alguns parâmetros genéticos.

\section{MATERIAL E MÉTODOS}

Os trabalhos experimentais com a lavoura da cana-de-açúcar (Saccharum spp.) foram desenvolvidos durante as safras agrícolas de 1999/2000, 2000/2001, 2001/2002 e 2002/2003, na área agrícola da Usina Olho D'Água, localizada na Zona Canavieira da Mata Norte de Pernambuco, no Município de Camutanga $\left(07^{\circ} 24^{\prime} \mathrm{S}\right.$ e $\left.35^{\circ} 28^{\prime} \mathrm{W}\right)$. O delineamento experimental foi o de blocos casualizados, com quatro blocos, utilizando-se como tratamentos dezesseis genótipos (Tabela 1), sendo quatro deles testemunhas (RB72454, RB813804, SP70-1143 e SP79-1011), consideradas padrões varietais. A unidade experimental foi representada por cinco sulcos de $8,0 \mathrm{~m}$ de comprimento, espaçados de $1,0 \mathrm{~m}$, contendo uma área útil de $40 \mathrm{~m}^{2}$.

Durante o período dos experimentos foram observados $2.107 \mathrm{~mm}, 959,5 \mathrm{~mm}, 1.285,5 \mathrm{~mm}$ e 1.418 $\mathrm{mm}$, de precipitações pluviais, respectivamente, para o $1 .^{\circ}, 2 .^{\circ}, 3 .^{\circ}$ e $4 .^{\circ}$ ciclos de colheita.

Os experimentos foram colhidos com média de 15 meses de idade para cana-planta, enquanto os de socaria estavam com 12 meses para os três cortes sucessivos. Foram determinados os valores para TCH (toneladas de cana por hectare), calculada 
por meio da transformação da massa da parcela em tonelada por hectare e TPH (toneladas de açúcar provável por hectare), multiplicando-se o TCH pelo percentual de sacarose aparente (PC). Para caracterização da qualidade tecnológica, determinaram-se o teor de fibra (FI), os valores de brix (PB), pol \% da cana (PC) e pureza (PZA), mediante amostras compostas por dez canas.

Os dados foram submetidos à análise genético-estatística individual e de grupos de experimentos. As análises individuais foram realizadas de acordo com Gomes (1990) utilizando o modelo matemático aditivo linear $Y_{i j}=\mu+g_{i}+b_{j}+a ̊ i j$, sendo, $Y_{\mathrm{ij}}$ : a observação do i-ésimo genótipo no j-ésimo bloco; $\mu$ : a média geral; $g_{i}$ : o efeito do i-ésimo genótipo; $\mathrm{b}_{\mathrm{j}}$ : o efeito do j-ésimo bloco; $\varepsilon_{\mathrm{ij}}$ : o componente aleatório. Nas análises de grupos de experimentos (Tabela 2) foi utilizado modelo proposto por YATES e COCHRAN (1938). A análise de variância de grupos de experimentos foi realizada segundo o modelo estatístico $Y_{i j k}=\mu+(b /$ $a)_{j k}+g_{i}+c_{k}+g c_{i j}+\varepsilon{ }_{i j k}$, sendo, $Y_{i j k}$ : o i-ésimo genótipo, no j-ésimo bloco dentro do k-ésimo corte; $\mu$ : a média geral do ensaio; $(b / c)_{j k}$ : o efeito do j-ésimo bloco dentro do k-ésimo corte; $\mathrm{g}_{\mathrm{i}}$ : o efeito do i-ésimo genótipo; $\mathrm{c}_{\mathrm{k}}$ : o efeito do k-ésimo corte; $\mathrm{gc}_{\mathrm{ij}}$ : o efeito da interação do i-ésimo genótipo com k-ésimo corte; $\varepsilon_{\mathrm{ijk}}$ : o efeito do erro experimental. Foram determinados como fixos, os efeitos do genótipo $(\mathrm{g})$, e aleatórios os efeitos do bloco (b) e corte (c). No caso da análise de grupos de experimento foram obedecidas as exigências de homogeneidade das variâncias, sendo o valor calculado do teste de Hartley menor que o trabalho.

As comparações de médias da produtividade agrícola, industrial e caracteres industriais foram formuladas por meio do teste de Tukey, ao nível de $1 \%$ de probabilidade. No caso de interação significativa entre genótipo $\mathrm{x}$ ambiente (corte), na análise de grupos de experimento, o quadrado médio residual adotado para comparações de tratamentos foi da interação entre genótipos x cortes.

Os cálculos dos componentes de variação fenotípica, genética, ambiental e interação genótipos $x$ cortes para todas as variáveis TCH (toneladas de canas por hectare), TPH (toneladas de açúcar provável por hectare), teor de fibra (FI), percentagem de brix (PB), pol \% da cana (PC) e pureza (PZA) foram efetuados conforme CRUZ (2001). Os coeficientes de variação genética, fenotípica, ambiental e da herdabilidade média, foram determinados segundo o estabelecido por VencovsKy (1987). As análises genético-estatísticas foram processadas através do programa GENES (CRUZ 2001).

Tabela 1. Identificação dos genótipos de cana-de-açúcar utilizados nos experimentos, genitores e procedência. Recife (PE), 2004

\begin{tabular}{|c|c|c|c|c|}
\hline \multirow{2}{*}{ Genótipos } & & \multicolumn{2}{|c|}{ Genitor } & \multirow{2}{*}{ Origem } \\
\hline & & Feminino & Masculino & \\
\hline 1 & RB 72454 & CP 53-76 & $?$ & RIDESA \\
\hline 2 & RB 813804 & CP 48-124 & $?$ & RIDESA \\
\hline 3 & RB 942724 & RB 83100 & $?$ & RIDESA \\
\hline 4 & RB 942991 & RB 955106 & $?$ & RIDESA \\
\hline 5 & RB 943016 & H 64-225 & $?$ & RIDESA \\
\hline 6 & RB 943047 & RB 855106 & $?$ & RIDESA \\
\hline 7 & RB 943052 & RB 7960 & $?$ & RIDESA \\
\hline 8 & RB 943066 & CP 57-621 & $?$ & RIDESA \\
\hline 9 & RB 943112 & Co 678 & $?$ & RIDESA \\
\hline 10 & RB 943163 & M 147-44 & $?$ & RIDESA \\
\hline 11 & RB 943176 & RB 75126 & RB 765418 & RIDESA \\
\hline 12 & RB 943263 & CB 41-115 & $?$ & RIDESA \\
\hline 13 & RB 943339 & ROC 3 & RB 83100 & RIDESA \\
\hline 14 & RB 943352 & IAC 48-65 & $?$ & RIDESA \\
\hline 15 & SP 70-1143 & IAC 48-65 & $?$ & COPERSUCAR \\
\hline 16 & SP 79-1011 & NA 56-79 & Co 775 & COPERSUCAR \\
\hline
\end{tabular}


Tabela 2. Esquema da análise de variância para a análise de grupos de experimentos com as respectivas esperanças de quadrados médios $\mathrm{E}(\mathrm{QM})$

\begin{tabular}{|c|c|c|c|c|}
\hline $\mathrm{FV}$ & GL & Q & $\mathrm{E}(\mathrm{QM})$ & $\mathrm{F}$ \\
\hline Blocos/Corte & $a(b-1)$ & $\mathrm{Q}_{1}$ & $\hat{\sigma}_{e}^{2}+\mathrm{g} \hat{\sigma}^{2} \mathrm{~b} / \mathrm{c}$ & $\mathrm{Q}_{1} / \mathrm{Q}_{5}$ \\
\hline Cortes (C) & $\mathrm{a}-1$ & $\mathrm{Q}_{2}$ & $\hat{\sigma}_{e}^{2}+\hat{\sigma}^{2} \mathrm{~g} \mathrm{~b} / \mathrm{c}+\mathrm{bg} \hat{\sigma} 2 \mathrm{a}$ & $\mathrm{Q}_{2} / \mathrm{Q}_{1}$ \\
\hline Genótipos (G) & $g-1$ & $\mathrm{Q}_{3}$ & $\hat{\sigma_{e}^{2}}+\operatorname{bg} \frac{\hat{\sigma}_{g a}^{2}}{g-1}+$ ba $\frac{\Sigma g_{i}^{2}}{g-1}$ & $\mathrm{Q}_{3} / \mathrm{Q}_{4}$ \\
\hline Interação $\mathrm{G} \times \mathrm{C}$ & $(\mathrm{a}-1)(\mathrm{g}-1)$ & $\mathrm{Q}_{4}$ & $\hat{\sigma}_{e}^{2}+\operatorname{bg} \frac{\hat{\sigma}_{g a}^{2}}{g-1}$ & $\mathrm{Q}_{4} / \mathrm{Q}_{5}$ \\
\hline Resíduo & $\mathrm{a}(\mathrm{a}-1)(\mathrm{g}-1)$ & $\mathrm{Q}_{5}$ & $\hat{\sigma_{e}^{2}}$ & \\
\hline Total & abg-1 & & & \\
\hline
\end{tabular}

\section{RESULTADOS E DISCUSSÃO}

Pelo teste F, foram detectadas diferenças altamente significativas $(\mathrm{P}<0,01)$ entre os genótipos para as variáveis toneladas de açúcar provável por hectare (TPH) e toneladas de canas por hectare (TCH) nos quatros cortes da cana e para o pol \% da cana (PC), teor de fibra (FI), pureza (PZA) e percentagem de brix (PB) apenas nos três primeiros cortes da cana (Tabela 3). Essa significância para TCH e TPH e caracteres industriais evidenciou a existência de alto grau de variabilidade genética entre os genótipos testados. Em estudos de famílias de cana-de-açúcar Bressiani (2001), Bressiani et al. (2002) e Silva et al. (2002a), constataram diferenças significativas $(p<0,01)$ para os caracteres TCH e TPH. BASTOS (1984), estudando o comportamento de variedades de canade-açúcar no Estado de Pernambuco verificou variabilidade fenotípica bastante significativa, o que favorece a obtenção de resultados positivos na escolha de genótipos de maior produtividade agrícola e industrial.

$\mathrm{Na}$ tabela 3, verifica-se que o parâmetro TCH foi de 91 e $48 \mathrm{t} /$ ha, respectivamente, para o $1 .^{\circ}$ e $2 .^{\circ}$ corte da cana. A redução observada n. $^{\circ} 2^{\circ}$ ciclo de corte pode ser atribuída à baixa precipitação pluvial ocorrida no período da safra de 2000/2001. Quanto ao TPH, o valor foi de 12,14 no $1 .^{\circ}$ corte, decrescendo para $5,88,6,30$ e 8,17 no $2 .^{\circ}$ e $3 .^{\circ}$ e $4 .^{\circ}$ cortes, respectivamente. As demais variáveis (PC, FI, PZA e $\mathrm{PB})$ apresentaram valores médios com pequena amplitude de variação nos quatro ciclos de colheita.

As variâncias genéticas distribuíram-se numa ampla faixa de valores, oscilando de 0,100 a 87,622 , sendo a menor para PC no $4 .^{\circ}$ corte e a maior para
$\mathrm{TCH}$ no 1 . $^{\circ}$ corte. A produção de cana $(\mathrm{TCH})$ nos quatro cortes apresentou os maiores valores para as variâncias genéticas. Com relação ao coeficiente de herdabilidade média $\left(\mathrm{h}^{2} \mathrm{~m}\right)$, observa-se que oscilaram de $94,6 \%$ a $5,1 \%$, respectivamente para $\mathrm{TCH}$ e $\mathrm{PB}$. Entretanto, os valores de herdabilidade média $\left(h^{2} \mathrm{~m}\right)$ foram expressivos para a produção de cana por hectare $(\mathrm{TCH})$ e toneladas de açúcar provável por hectare (TPH) em todos os quatro cortes da cana. Essa evidência sugere a possibilidade de progresso com a seleção desses caracteres, atribuída à existência de variabilidade genética inerente entre os genótipos testados, certamente em razão de cada uma delas constituir uma identidade genética distinta (Tabela 3). Os coeficientes de variação foram baixos oscilando entre $2,01 \%$ e $11,51 \%$, indicando boa precisão experimental.

Os resultados exibidos na tabela 4 para o teste $F$ revelam diferenças altamente significativas entre os genótipos (G), os ambientes (cortes) e para interação genótipo $x$ corte $(\mathrm{G} \times \mathrm{C})$, para os caracteres $\mathrm{TCH}, \mathrm{TPH}$, FI e PZA, ao nível de $1 \%$ e para $\mathrm{PC}$ e $\mathrm{PB}, 5 \%$ de probabilidade, evidenciando a existência de alto grau de variabilidade genética entre os genótipos de canade-açúcar. Essas evidências podem assegurar ao melhorista aplicação, nos genótipos em questão de métodos de melhoramento adequados com perspectivas favoráveis de seleção de plantas promissoras. A variância significativa da interação (G x C) para variável pureza (PZA), não é concordante com os resultados obtidos por Moura (1990). Apesar da variância significativa da interação $(G \times C)$ para o teor de fibra (FI) diferir dos resultados de LANDELL et al. (1999), a variância significativa para o brix é concordante com os trabalhos de SiLva et al. (2002b). 
A respeito da interação significativa com o ambiente, pelos resultados observa-se que todos os caracteres foram altamente influenciados pelos cortes (ciclo da cana), tendo a variável TCH exibido valor em magnitude superior aos demais caracteres, mostrando ser a variável mais influenciada pelos ciclos de colheita da cana (Tabela 4). Resultados semelhantes foram observados por LANDELL et al. (1999) e SANTOS (2004). Quanto à significância da interação genótipo $\mathrm{x}$ corte para as variáveis $\mathrm{TCH}$, TPH, PC, FI, PZA e PB, verifica-se que os cortes representam ambientes contrastantes, ou seja, os genótipos avaliados têm comportamento dependente do corte (ciclo de colheita). Os estudos de LANDELL et al. (1999) mostraram resultados semelhantes apenas para TCH, TPH e PC. A análise de variância de grupos de experimento indicou diferenças altamente significativas em relação às fontes de variação de genótipos, ambiente (corte) e da interação entre eles. Esse fato significa que os genótipos testados diferiram para as variáveis estudadas, bem como o ambiente (cortes) por causa dos fatores edáficos e climáticos dos anos ou ciclos da cultura, em que os trabalhos foram desenvolvidos. Rosse et al. (2002), estudando a comparação de métodos de regressão para avaliar a estabilidade fenotípica em cana-de-açúcar, encontraram resultados semelhantes.

Tabela 3. Resumo da análise de variância em cada corte, para as variáveis toneladas de cana por hectare (TCH), tonelada de açúcar por hectare (TPH), pol \% corrigido (PC), fibra (FI), pureza (PZA) e brix (PB) na Região da Mata Norte de Pernambuco, Recife, 2004

\begin{tabular}{|c|c|c|c|c|c|c|c|}
\hline \multirow{2}{*}{ Cortes } & \multirow{2}{*}{ Variáveis } & \multicolumn{2}{|c|}{ Quadrado médio } & \multirow{2}{*}{ Média geral } & \multirow{2}{*}{ CV (\%) } & \multirow{2}{*}{$(\hat{\sigma} 2 \mathrm{~g})$} & \multirow{2}{*}{$\mathrm{h}^{2} \mathrm{~m}(\%)$} \\
\hline & & Genótipos & Resíduo & & & & \\
\hline & $\mathrm{TCH}$ & $373,482292^{* *}$ & 22,990625 & 91,23 & 5,26 & 87,622 & 93,8 \\
\hline & $\mathrm{TPH}$ & $9,602382^{* *}$ & 0,742862 & 12,14 & 7,09 & 2,215 & 92,3 \\
\hline $1^{\circ}$ corte & PC & $2,789056^{* *}$ & 0,571360 & 13,33 & 5,67 & 0,554 & 79,5 \\
\hline \multirow[t]{5}{*}{2000} & FI & $6,98979 * *$ & 0,694284 & 11,82 & 7,05 & 1,574 & 90,1 \\
\hline & PZA & $13,464821^{* *}$ & 5,243613 & 86,03 & 2,66 & 2,055 & 61,1 \\
\hline & PB & $3,084958^{* *}$ & 0,747876 & 18,23 & 4,74 & 0,584 & 75,8 \\
\hline & $\mathrm{TCH}$ & $153,633333^{* *}$ & 8,355556 & 48,25 & 5,99 & 36,319 & 94,6 \\
\hline & ТPH & $2,665095^{* *}$ & 0,203300 & 5,88 & 7,67 & 0,615 & 92,4 \\
\hline $2^{\circ}$ corte & PC & $1,556778^{* *}$ & 0,539764 & 12,19 & 6,03 & 0,254 & 65,3 \\
\hline \multirow[t]{5}{*}{2001} & FI & $11,77411^{* *}$ & 0,833064 & 15,84 & 5,76 & 2,735 & 92,9 \\
\hline & PZA & $9,756136^{* *}$ & 2,992378 & 86,02 & 2,01 & 1,691 & 69,3 \\
\hline & PB & $3,225323^{* *}$ & 0,898767 & 17,91 & 5,29 & 0,581 & 72,1 \\
\hline & $\mathrm{TCH}$ & $126,783333^{* *}$ & 10,747222 & 57,81 & 5,67 & 29,009 & 91,5 \\
\hline & $\mathrm{TPH}$ & $3,615964^{* *}$ & 0,344647 & 6,30 & 9,31 & 0,818 & 90,5 \\
\hline $3^{\circ}$ corte & PC & $3,766089^{* *}$ & 0,714811 & 10,88 & 7,77 & 0,763 & 81,0 \\
\hline \multirow[t]{5}{*}{2002} & FI & $10,53019^{* *}$ & 0,857864 & 13,20 & 7,02 & 2,418 & 91,9 \\
\hline & PZA & $29,09720^{* *}$ & 10,413862 & 84,59 & 3,82 & 4,671 & 64,2 \\
\hline & PB & $7,067500^{* *}$ & 0,871056 & 15,51 & 6,02 & 1,549 & 87,7 \\
\hline & $\mathrm{TCH}$ & $320,290625^{* *}$ & 31,507292 & 65,58 & 8,56 & 72,196 & 90,2 \\
\hline & $\mathrm{TPH}$ & $6,330943^{* *}$ & 0,657027 & 8,17 & 9,92 & 1,418 & 89,6 \\
\hline $4^{\circ}$ corte & PC & $1,486900 \mathrm{NS}$ & 1,085253 & 12,46 & 8,36 & 0,100 & 27,0 \\
\hline \multirow[t]{3}{*}{2003} & FI & 3,388444 NS & 2,177502 & 12,81 & 11,51 & 0,303 & 35,7 \\
\hline & PZA & $6,717306 \mathrm{NS}$ & 4,964216 & 87,37 & 2,55 & 0,438 & 26,1 \\
\hline & $\mathrm{PB}$ & $1,714740 \mathrm{NS}$ & 1,627433 & 17,08 & 7,47 & 0,022 & 5,1 \\
\hline
\end{tabular}

** Significativo ao nível de $1 \%$ pelo teste F.

NS: Não significativo. 
Tabela 4. Resultado da análise de variância dos caracteres tonelada de cana por hectare (TCH), tonelada de açúcar por hectare $(\mathrm{TPH})$, pol \% corrigido (PC), fibra (FI), pureza (PZA) brix (PB) e a relação do maior quadrado médio do resíduo (QMR) e o menor (teste de Hartley), avaliados em análise de grupos de experimento do experimento na Região da Mata Norte Pernambuco, Recife, 2004

\begin{tabular}{|c|c|c|c|c|c|c|c|}
\hline \multirow{2}{*}{ F.V } & \multirow{2}{*}{ G.L } & \multicolumn{6}{|c|}{ Quadrados médios } \\
\hline & & $\mathrm{TCH}$ & $\mathrm{TPH}$ & PC & FI & PZA & $\mathrm{PB}$ \\
\hline Genótipos & 15 & $656,658^{* *}$ & $16,586^{* *}$ & $6,181^{* *}$ & $22,315^{* *}$ & $26,001^{*}$ & $10,069^{* *}$ \\
\hline Cortes & 3 & $21732,969 * *$ & $524,519^{* *}$ & $65,992^{* *}$ & $188,628^{* *}$ & $82,487^{* *}$ & $94,812^{* *}$ \\
\hline Genótipo x Corte & 45 & $105,844^{* *}$ & $1,877^{* *}$ & $1,139 *$ & $3,456^{* *}$ & $11,009^{* *}$ & $1,674^{*}$ \\
\hline Resíduo & 180 & 18,400 & 0,487 & 0,728 & 1,141 & 5,904 & 1,036 \\
\hline Médias & - & 65,72 & 8,12 & 12,21 & 13,42 & 86,00 & 17,18 \\
\hline C.V $(\%)$ & - & 6,53 & 8,59 & 6,94 & 7,96 & 2,83 & 5,92 \\
\hline$>(\mathrm{QMR}) /<(\mathrm{QMR})$ & - & 3,77 & 3,65 & 2,01 & 3,14 & 3,48 & 2,18 \\
\hline
\end{tabular}

**; * :Significativo ao nível de $1 \%$ e 5\% pelo teste $\mathrm{F}$ respectivamente.

Observa-se ainda na tabela 4 que a produção de cana por hectare $(\mathrm{TCH})$ exibiu valores mais altos em magnitude para a interação genótipo $x$ corte $(G x$ C). Verificou-se que na decomposição de TPH em TCH e PC na fonte de variação da interação $(\mathrm{G} \times \mathrm{C}), \mathrm{o} \mathrm{TCH}$ foi o que mais se destacou com proporção de 1:41 para o TCH, e o PC com aproximadamente 1:8. Com base nesses resultados, pode-se concluir que a interação ( $G$ $x \mathrm{C}$ ) exerce mais influência sobre o TCH e menos sobre o PC, confirmando a resposta específica de genótipos para essa variável a ambientes diferenciados. Os estudos em cana-de-açúcar realizados por LANDELL et al. (1999) e BRESSIANi (2001), revelaram valores de variância maiores para TCH do que para as variáveis teor de açúcar PC e TPH. Todavia, a importância significativa da interação genótipo $\mathrm{x}$ corte para os caracteres TCH e TPH foi identificada tanto ao nível de clones por Raizer e Vencovsky (1999), quanto ao nível de famílias por Bull et al. (1992) e Bissessur et al. (2000).

A produtividade em TCH e TPH entre as padrões RB72454, RB813804, SP70-1143 e SP79-1011 se equivaleram estatisticamente entre si, ao nível de $1 \%$. Entretanto, quanto ao TCH, a variedade RB813804 diferiu dos clones RB943052, RB942724, RB943047, RB943176, RB943066 e RB943263. Com relação ao TPH, a variedade RB 813804 diferiu, ao nível de 1\%, dos clones RB943352, RB943016, RB 943163, RB 943112, RB 943052, RB942724, RB943047, RB943176, RB943066 e RB943263 (Tabela 5). Com referência ao pol \% corrigido (PC), observa-se que as variedadespadrão não diferiram entre si, ao nível de $1 \%$, mas a RB813804 e SP70-1143 diferiram do clone RB943016. Verifica-se que a RB813804 se destaca com o valor mais alto de PC $(13,18 \%)$. Para FI, verifica-se que as variedades padrão também não diferiram estatisticamente entre si. Apenas os clones RB942991 e RB943163, diferiram da variedade padrão SP701143 , ao nível de $1 \%$ de probabilidade. Quanto ao PZA, as variedades padrões se equivaleram entre si. O padrão RB813804, com o valor de $88,62 \%$ diferiu estatisticamente do clone RB943352, com 83,23\%; este, por sua vez, diferiu da RB942724 que apresentou o valor $87,65 \%$, estatisticamente igual ao daquele padrão. A porcentagem de fibra (PB) das padrões RB813804 e SP70-1143 diferiu estatisticamente do clone RB943016.

As variâncias genéticas entre os genótipos distribuíram-se em ampla faixa de valores (Tabela 6), variando de 0,315 para o pol \% corrigido (PC) a 34,427 para a produção de cana por hectare (TCH). Observase pequena variação genética relativa à magnitude dos valores estimados para os caracteres TPH $(0,919)$, PC $(0,315)$, FI $(1,178)$, PZA $(0,937)$ e PB $(0,524)$. Considerando a importância genética, o TCH exibiu valor em magnitude superior as demais variáveis, refletindo maior variabilidade genética. Os resultados encontrados em cana-de-açúcar por SANTOS et al. (2004) foram semelhantes aos obtidos neste trabalho.

Quanto às estimativas das variâncias fenotípicas, os valores oscilaram de 1,042 a 52,827, sendo o menor para o PC e o maior para TCH. Quanto à variância da interação genótipo-ambiente, os valores variaram de 0,096, para PC, a 20,494, para TCH. A produção de cana por hectare $(\mathrm{TCH})$ na componente da interação genótipo x corte $\left(\hat{\sigma}^{2} \mathrm{gc}\right)$ foi elevada, confirmando a resposta específica de genótipos a ambientes diferenciados para esse caráter. LANDELL et al. (1999), estudando clones de cana-de-açúcar do Instituto Agronômico (IAC), de Campinas, em fase 
final de experimentação na região de Ribeirão Preto (SP), verificaram resultados semelhantes para a variável $\mathrm{TCH}$. Infere-se que a produção de cana por hectare é um dos caracteres da cana-de-açúcar mais influenciado pela interação genótipo x ambiente. Assim, os clones e variedades-padrão refletiram comportamento diferentes durante os ciclos de corte da cana.

Nas estimativas de variância fenotípica $\left(\hat{\sigma}^{2} \mathrm{f}\right)$, genética $\left(\hat{\sigma}^{2} \mathrm{~g}\right)$ e da componente da interação genótipo ambiente $\left(\hat{\sigma}^{2} \mathrm{gc}\right)$, verificaram-se as maiores magnitudes para a produção de cana por hectare (TCH), com valores de 52,827, 34,427 e 20,494 respectivamente. Essa variabilidade pode ser atribuída às diferenças existentes entre os genótipos estudados para esse caráter.

Os coeficientes de variação genética (Tabela 6), indicadores da quantidade de variabilidade genética existente entre os genótipos estudados quanto às médias populacionais, variaram de $1,25 \%$ a $11,80 \%$. Os coeficientes de variação genética foram mais elevados em magnitude para variáveis TPH, TCH e FI, sendo também maiores do que os coeficientes de variação ambiental. Com base nesses resultados sugere-se que tais genótipos sejam estudados em outros ambientes para que se possa detectar vantagem competitiva de alguns desses clones, para algumas das características avaliadas. Em programas de melhoramento, pode-se recomendar a utilização para seleção das variáveis estudadas. Ressalte-se que seja feita uma escolha com critérios mais apropriados de genitores e de cruzamentos para obtenção de populações com maiores perspectivas de ganhos genéticos. Avaliando genótipos de cana-de-açúcar LANDELL et al. (1999), observaram valores maiores de coeficientes de variação genética para as variáveis $\mathrm{TPH}(12,87 \%)$ e TCH $(14,06 \%)$, fato que se assemelha ao do presente estudo.

Tabela 5. Valores médios dos caracteres tonelada de cana por hectare (TCH), tonelada de açúcar por hectare (TPH), pol \% corrigido (PC), fibra (FI), pureza (PZA) e brix (PB), avaliados em análise de grupos de experimento na Região da Mata Norte de Pernambuco, Recife, 2004

\begin{tabular}{|c|c|c|c|c|c|c|}
\hline \multirow{2}{*}{ Clones } & \multicolumn{6}{|c|}{ Caracteres } \\
\hline & $\mathrm{TCH}$ t cana/ha & TPH t pol/ha & PC \% & FI \% & PZA \% & PB \% \\
\hline 1. RB813804* & $76,88 \mathrm{a}$ & $10,29 \mathrm{a}$ & $13,18 \mathrm{a}$ & 13,34 abcde & 88,62 a & $17,97 \mathrm{abc}$ \\
\hline 2. SP79-1011* & $73,09 \mathrm{ab}$ & $9,13 \mathrm{abc}$ & $12,23 \mathrm{abcd}$ & 14,20 abcd & $85,54 \mathrm{ab}$ & 17,52 abcde \\
\hline 3. RB942991 & $72,38 \mathrm{abc}$ & $9,37 \mathrm{ab}$ & $12,81 \mathrm{ab}$ & $11,91 \mathrm{de}$ & $85,41 \mathrm{ab}$ & 17,68 abcd \\
\hline 4. RB943339 & $71,25 \mathrm{abcd}$ & $8,74 \mathrm{abcd}$ & $12,17 \mathrm{abcd}$ & $13,71 \mathrm{abcd}$ & $87,05 \mathrm{ab}$ & 17,01 bcde \\
\hline 5. RB72454* & 70,07 abcd & 8,65 abcd & $12,21 \mathrm{abcd}$ & 12,72 bcde & $86,28 \mathrm{ab}$ & 16,90 bcde \\
\hline 6. SP70-1143* & $69,19 \mathrm{abcd}$ & $8,97 \mathrm{abc}$ & $12,94 \mathrm{a}$ & $14,44 \mathrm{abc}$ & $86,38 \mathrm{ab}$ & $18,48 \mathrm{ab}$ \\
\hline 7. RB943352 & $69,00 \mathrm{abcd}$ & 8,25 bcde & $11,94 \mathrm{abcd}$ & 12,20 cde & $83,23 \mathrm{~b}$ & 16,98 bcde \\
\hline 8. RB943016 & 65,63 abcde & 7,44 cdef & $11,20 \mathrm{~d}$ & 13,89 abcd & $85,97 \mathrm{ab}$ & $15,91 \mathrm{e}$ \\
\hline 9. RB943163 & 65,38 abcde & 8,02 bcdef & 13,12 abcd & $11,00 \mathrm{e}$ & $85,53 \mathrm{ab}$ & 16,47 cde \\
\hline 10. RB943112 & 64,81 abcde & 7,60 cdef & 11,52 bcd & $14,26 \mathrm{abcd}$ & $84,45 \mathrm{ab}$ & 16,72 cde \\
\hline 11. RB943052 & 60,94 bcde & 7,72 bcdef & $12,52 \mathrm{abcd}$ & $13,94 \mathrm{abcd}$ & $86,54 \mathrm{ab}$ & 17,66 abcd \\
\hline 12. RB942724 & 60,25 cde & 7,69 bcdef & $12,62 \mathrm{abc}$ & 12,67 bcde & $87,65 \mathrm{a}$ & 17,17 abcde \\
\hline 13. RB943047 & $60,13 \mathrm{cde}$ & 7,08 def & $11,52 \mathrm{bcd}$ & 12,13 cde & $84,69 \mathrm{ab}$ & $16,06 \mathrm{de}$ \\
\hline 14. RB943176 & $58,94 \mathrm{de}$ & 7,79 bcdef & $13,17 \mathrm{a}$ & $14,38 \mathrm{abc}$ & $86,60 \mathrm{ab}$ & $18,71 \mathrm{a}$ \\
\hline 15. RB943066 & $58,75 \mathrm{de}$ & 6,81 ef & $11,43 \mathrm{~cd}$ & $14,77 \mathrm{ab}$ & $85,70 \mathrm{ab}$ & 16,50 cde \\
\hline 16. RB943263 & $54,25 \mathrm{e}$ & $6,43 \mathrm{f}$ & 11,86 abcd & $15,13 \mathrm{a}$ & $86,34 \mathrm{ab}$ & 17,18 abcde \\
\hline Médias ambientes & 65,72 & 8,12 & 12,21 & 13,42 & 86,00 & 17,18 \\
\hline Médias padrões & 72,30 & 9,26 & 12,64 & 13,67 & 86,70 & 17,71 \\
\hline DMS (1\%) & 13,187 & 1,756 & 1,368 & 2,383 & 4,253 & 1,659 \\
\hline
\end{tabular}

Médias seguidas da mesma letra não diferem estatisticamente, ao nível de 1\% pelo teste de Tukey.

* Variedades-padrão. 
Tabela 6. Estimativas de parâmetros associados às variâncias fenotípica $\left(\hat{\sigma}^{2} \mathrm{f}\right)$, genética $\left(\hat{\sigma}^{2} \mathrm{~g}\right)$, interação genótipo ambiente $(\hat{\sigma} 2 \mathrm{gc})$, herdabilidade média $(\mathrm{h} 2 \mathrm{~m})$, coeficiente de variação genético $\left(\mathrm{CV}_{\mathrm{g}}\right)$, coeficiente de variação ambiental $\left(\mathrm{CV}_{\mathrm{a}}\right)$ e da razão $\left(\mathrm{CV}_{\mathrm{g}} / \mathrm{CV}_{\mathrm{a}}\right)$ dos caracteres tonelada de cana por hectare $(\mathrm{TCH})$, tonelada de pol por hectare (TPH), pol \% corrigido (PC), fibra (FI), pureza (PZA) e brix (PB), avaliados em análise de grupos de experimento na Região da Mata Norte de Pernambuco, Recife, 2004

\begin{tabular}{lccccccc}
\hline \multirow{2}{*}{ Caracteres } & \multicolumn{7}{c}{ Parâmetros } \\
\cline { 2 - 7 } & $\hat{\sigma}^{2} \mathrm{f}$ & $\hat{\sigma}^{2} \mathrm{~g}$ & $\hat{\sigma}^{2} \mathrm{gxc}$ & $\mathrm{CV}_{\mathrm{g}}(\%)$ & $\mathrm{CV}_{\mathrm{a}}(\%)$ & $\mathrm{CV}_{\mathrm{g}} / \mathrm{CV}_{\mathrm{a}}$ & $\mathrm{h}^{2} \mathrm{~m}(\%)$ \\
\hline TCH & 52,827 & 34,427 & 20,494 & 8,930 & 6,527 & 1,472 & 83,8 \\
TPH & 1,405 & 0,919 & 0,326 & 11,800 & 8,580 & 1,437 & 88,7 \\
PC & 1,042 & 0,315 & 0,096 & 4,595 & 6,980 & 0,683 & 81,5 \\
FI & 2,318 & 1,178 & 0,542 & 8,091 & 7,958 & 1,077 & 84,5 \\
PZA & 6,840 & 0,937 & 1,196 & 1,125 & 2,825 & 0,461 & 57,6 \\
PB & 1,560 & 0,524 & 0,149 & 4,215 & 5,924 & 0,737 & 83,3 \\
\hline
\end{tabular}

Os coeficientes de herdabilidade média $\left(\mathrm{h}^{2} \mathrm{~m}\right)$ variaram de $57,6 \%$ a $88,7 \%$, respectivamente, para PZA e TPH. O percentual de pureza (PZA) foi de valor relativamente mais baixo de herdabilidade média (57,6\%). Cruz e Regazzi (1997) afirmam que os caracteres complexos comumente têm baixa herdabilidade, enquanto os caracteres menos complexos ostentam valores mais elevados desse parâmetro. As variáveis TCH, TPH, PC FI e PB exibiram valores de herdabilidade média superior a $80 \%$. LANDELL et al. (1999), estudando clones de canade-açúcar em três ambientes com quatro cortes em fase final de seleção, notaram valores altos de herdabilidade média diferentes a este trabalho para as variáveis TCH de $(98,94 \%)$ e TPH de $(99,01 \%)$. No caso do brix, SILVA et al. (2002a) observaram valores de herdabilidade em planta individual de $50 \%$ e de 90\% para famílias em cana-de-açúcar.

\section{CONCLUSÕES}

1. As variedades padrões RB813804, RB72454, SP79-1011 e SP70-1143, confirmaram o potencial em produção de cana por hectare e de açúcar por hectare nas condições ambientais da Zona da Mata Norte de Pernambuco.

2. O efeito de corte da cana foi altamente significativo, indicando o comportamento específico entre os genótipos nos diversos cortes da cana.

3. A tonelada de cana por hectare foi o componente mais influenciado pelos ciclos de colheita da cana.
4. Sugere-se que o clone RB942991 seja testado novamente para o TCH, TPH, PC, FI, PZA e PB em outros ambientes das Zonas da Mata de Pernambuco.

\section{REFERÊNCIAS}

BASSINELO, A. I.; ABRAHÃO, I. S.; VALADÃO, M. B.; BARCELLOS, J. E. T.; PICCOLO, C. R. Primeiros resultados de estudos de novas variedades de cana-de-açúcar em solos de cerrado. In: CONGRESSO NACIONAL DA STAB, 3., e CONVENÇÃO DA ACTALAC, 5., 1984, São Paulo. Anais... São Paulo: STAB, 1984. p. 206-214.

BASTOS, G. Q. Análise conjunta de competições varietais de cana-de-açúcar (Saccharum spp.) com dois tratamentos comuns, no Estado de Pernambuco. 1984. 121f. Dissertação (Mestrado) - Universidade Federal Rural de Pernambuco, Recife.

BISSESSUR, D.; TILNEY-BASSETT; R.A.E.; LIMSHIN CHONG, L.C.Y.; DOMAINGUE, R.; JULIEN, M.H.R. Family x environment and genotype $\mathrm{x}$ environment interactions for sugarcane across two contrasting marginal environments in Mauritius. Experimental Agriculture, Great Britain, v.36, p.101-114, 2000.

BRESSIANI, J. A. Interação entre famílias de cana-de-açúcar e locais: efeito na resposta esperada com a seleção. Bragantia, Campinas, v.61, n.1., 2002.

BRESSIANI, J. A. Seleção seqüencial em cana-de-açúcar. 2001. 133p. Tese (Doutorado). Escola Superior de Agricultura Luiz de Queiroz. Universidade de São Paulo, Piracicaba.

BULL, J.K.; HOGARTH, D.M.; BASFORD, K.E. Impact of genotype $x$ environment interactions on response to selection in sugarcane. Australian Journal of Experimental Agriculture, Melbourne, v.32, p.731-737, 1992. 
CRUZ, C.D. Programa GENES: versão Windows. Aplicativo computacional em genética e estatística. Viçosa: Editora UFV, 2001. 648p.

CRUZ, C.D.; REGAZZI, A.J. Modelos biométricos aplicados ao melhoramento genético. $2^{a}$ ed. rev. Viçosa: Editora UFV, 1997. 390p.

DIAS, F.L.F. Relação entre a produtividade, clima, solos e variedades de cana-de-açúcar, na Região Noroeste do Estado de São Paulo. Piracicaba, 1997. 64f. Dissertação (Mestrado). Escola Superior de Agricultura "Luiz de Queiroz" Universidade de São Paulo, Piracicaba.

FALCONER, D. S.; MACKAY, T.F.C. Introduction to quantitative genetics. 4.ed. Longman: Malaysia, 1996. 464 p.

FARIAS, S. O. Adaptabilidade e estabilidade de clones de canade-açúcar (Saccharum spp.) para a produção de pol e de biomassa no Nordeste do Brasil. 1992. 132p. Dissertação (Mestrado) - Universidade Federal Rural de Pernambuco.

GHELLER, A.C.A. GARCIA, A.A.F.; MENDES, J.M. Variedades RB: Comportamento de variedades comerciais e clones promissores na Região Norte do Estado de São Paulo, em três épocas de colheita. In: CONGRESSO NACIONAL DA SOCIEDADE DOS TÉCNICOS AÇUCAREIRO E ALCOOLEIROS DO BRASIL - STAB, 6., 1996, Maceió. Anais... Maceió:STAB, 1996. p.181-187.

GOMES, F.P. In: Curso de estatística experimental. 12.ed. Piracicaba: ESALQ, 1990, 468p.

LANDELL, M. G. A.; ALVAREZ R.;ZIMBACK, L.; CAMPANA M. P., SILA, M. A.; PEREIRA, J. C. V. N. A.; PERECIN D.; GALLO, P. B.; MARTINS, A. L. M.; KANTHACK, A.; FIGUEIREDO P.; VASCONCELOS C. M. Avaliação final de clones IAC de canade-açúcar da série 1982, em Latossolo Roxo da Região de Ribeirão Preto. Bragantia, Campinas, v. 58, n.2, p.1-13, 1999.

MOURA, M. M. Estimativas de parâmetros genéticos de caracteres industriais de híbridos de Cana-de-açúcar. 1990. $136 f$. Dissertação (Mestrado) - Universidade Federal Rural de Pernambuco, Recife.
RAIZER, A.J.; VENCOVSKY, R. Estabilidade fenotípica de novas variedades de cana-de-açúcar para o Estado de São Paulo. Pesquisa Agropecuária Brasileira, Brasília, v.34, n.12, p.2241-2246, 1999.

RAMALHO, M.A.P.; SANTOS, J.B.; PINTO, C.A.B.P. Genética na agropecuária. 2.ed. Lavras: UFLA, 2001. 472p.

ROSSE, L.N.; VENCOVSKY, R.; FERREIRA D. F. Comparação de métodos de regressão para avaliar a estabilidade fenotípica em cana-de-açúcar. Pesquisa Agropecuária Brasileira, Brasília, v.37, n.1, p.23-32, 2002.

SANTOS, M.S.M.; MADALENA, J.A.; SOARES, L.; FERREIRA, P.V.; BARBOSA, G.V.S. Repetibilidade de características agroindustriais em cana-de-açúcar. Pesquisa Agropecuária Brasileira, Brasília, v.39, n.4, p301-306, 2004.

SILVA, M.A.; GONÇALVES, P. S.; LANDELL, M. G. A.; BRESSIANI, A. J. Estimates of parameters and expected gains from selection of yield traits in sugarcane families. Crop Breeding and Applied Biotechnology, v.2, p.569578, 2002a.

SILVA, M.A.; LANDELL, M.G.A.; GONÇALVES, P.S.; MARTINS, A.L.M. Yield components in sugarcane families at four locations in the state of São Paulo, Brazil. Crop Breeding and Applied Biotechnology, v.2, n.1. p.97-106, 2002b.

SOUZA-VIEIRA, O.; MILLIGAN, S. B. Intrarow plant spacing and family $x$ environment interaction effects on sugarcane family evaluation. Crop Science, Madison, v.39, p.358-364, 1999.

VENCOVSKY, R. Herança quantitativa. In: PATERNIANI, E.; VIEGAS, G.P. (Ed.). Melhoramento e produção do milho no Brasil. 2.ed. Campinas: Fundação Cargill, 1987. Cap. 5, p.137-214.

YATES, F.; COCHRAN, W.G. The analysis of groups of experiments. The Journal of Agricultural Science, Cambridge, v. 28, n. 4 , p. $556-580,1938$. 